\title{
Evaluation of synthetic promoters in Physcomitrella patens
}

Peramuna, Anantha ; Bae, Hansol; Rasmussen, Erling Koch; Dueholm, Bjørn; Waibel, Thomas; Critchley, Joanna H.; Brzezek, Kerstin; Roberts, Michael; Simonsen, Henrik Toft

\section{Published in:}

Biochemical and Biophysical Research Communications

Link to article, DOI:

10.1016/j.bbrc.2018.04.092

Publication date:

2018

Document Version

Peer reviewed version

Link back to DTU Orbit

Citation (APA):

Peramuna, A., Bae, H., Rasmussen, E. K., Dueholm, B., Waibel, T., Critchley, J. H., Brzezek, K., Roberts, M., \& Simonsen, H. T. (2018). Evaluation of synthetic promoters in Physcomitrella patens. Biochemical and

Biophysical Research Communications, 500(2), 418-422. https://doi.org/10.1016/j.bbrc.2018.04.092

\section{General rights}

Copyright and moral rights for the publications made accessible in the public portal are retained by the authors and/or other copyright owners and it is a condition of accessing publications that users recognise and abide by the legal requirements associated with these rights.

- Users may download and print one copy of any publication from the public portal for the purpose of private study or research.

- You may not further distribute the material or use it for any profit-making activity or commercial gain

- You may freely distribute the URL identifying the publication in the public portal 


\section{Evaluation of synthetic promoters in 2 Physcomitrella patens}

4 Anantha Peramuna ${ }^{1}$, Hansol Bae ${ }^{1,2}$, Erling Koch Rasmussen ${ }^{1}$, Bjørn Dueholm ${ }^{1}$, Thomas Waibel ${ }^{3}$,

5 Joanna H. Critchley ${ }^{3}$, Kerstin Brzezek ${ }^{3}$, Michael Roberts ${ }^{3}$, Henrik Toft Simonsen ${ }^{1,2 *}$

6

$7 \quad{ }^{1}$ Department of Biotechnology and Biomedicine, Technical University of Denmark, Søltofts Plads

$8 \quad 223,2800$ Kgs. Lyngby, Denmark

$9 \quad{ }^{2}$ Mosspiration Biotech IVS, Hørsholm, Denmark

$10 \quad{ }^{3}$ Synpromics, Edinburgh, Scotland

11

$12 *$ hets@dtu.dk

Highlights

16

- Cis-elements can be randomly assembled to construct short plant promoters

- Synthetic promoters have higher mRNA expression level than the endogenous PpAct7

18

- Synthetic promoters have similar protein expression levels to AtUBQ10 promoter

19 


\section{Abstract}

22 Securing a molecular toolbox including diverse promoters is essential for genome engineering.

23 However, native promoters have limitations such as the available number or the length of the

24 promoter. In this work, three short synthetic promoters were characterized by using the yellow

25 fluorescent protein Venus. All of the tested promoters were active and showed mRNA activity higher

26 than housekeeping gene PpAct7, and similar protein expression level to AtUBQ10 promoter. This

27 study shows that few cis-elements are enough to establish a strong promoter for continuous

28 expression of genes in plants. Along with this study, enhance the number of available promotors to

29 be used in P. patens. It also demonstrate the potential to construct multiple non-native promoters on

30 demand, which would aid to resolve the bottleneck issue of multiple pathway expression in P. patens

31 and other plants.

33 Keywords: Physcomitrella patens; Venus; Ubiquitin promoter; Actin promoter; Synthetic Biology;

34 synthetic promoters 


\section{Introduction}

36 Genes in eukaryotes consist of three major regions, the promoter, the coding strand and the terminator. The promoter controls the gene expression by regulating the binding of transcription factors to recruit RNA polymerase (Latchman 1997). Thus, the synthesis of mRNA is directly correlated with the promoter activity that may lead to the production of protein. The promoter is situated thousands of base pairs (bp) upstream from the transcription start site (TSS), to about $30 \mathrm{bp}$ downstream from the TSS (Porto et al. 2014). Promoters come in different type of switches, some are constitutive, some react to specific stimuli, and some are inducible and maintain a strict on/off style switch.

Plants are known to have larger promoter sequences than those found in fungi and prokaryotes. Plant promoters typically range from $500 \mathrm{bp}$ to over 2,000 bp (Liu and Stewart 2016). As the possibilities in synthetic biology advances, it is often necessary to introduce multiple genes and promoters to achieve the desired traits. However, endogenous plant promoters are often of limited use in plant synthetic biology as multiple copies of the same promoter can trigger homology-dependent gene silencing (Halpin 2005). Therefore, characterizing multiple promoters from heterologous species has become important for fine-tuning of multiple genes.

Physcomitrella patens is a plant model system that has been used extensively to study plant evolution, physiology, and development (Vesty et al. 2016). The full genome is sequenced (Rensing et al. 2007) and development growth media and transformation methods are well described (Bach et al. 2014). Its ability to perform efficient homologues recombination, that now can be explained with RecQ helicase function (Wiedemann et al. 2018), is unique among plants enabling in vivo assembly of multiple DNA fragments followed by targeted genome integration by homologues recombination (King et al. 2016). Collectively, such distinct features make $P$. patens attractive as an industrial 
production platform for small natural products, which requires integration of numerous genes (Zhan et al. 2014; Ikram et al. 2015; Pan et al. 2015; Sabovljević et al. 2016; Khairul Ikram et al. 2017).

Monocot housekeeping gene promoters and the $35 \mathrm{~S}$ promoter from Cauliflower mosaic virus (CaMV) have shown a high-level of gene expression in P. patens (Horstmann et al. 2004; Saidi et al. 2005). However, the limited number of available promoters are a bottleneck in introducing complex pathways into $P$. patens and typically long plant promoters are difficult to handle in multiple gene integrations. Therefore, developing strong and short synthetic promoters has emerged as a major interest to provide a solution.

Synthetic promoters are relatively short (300-500 bp) and can be generated in countless number with similar strength or function, and could improve the genome stability (Roberts 2011). Cis-regulatory elements (CREs) of native promoters are non-conserved among genes that are similarly expressed. Thus, the synthetic promoters can be reasonably constructed to give high gene expression with a smaller size (Liu and Stewart 2016). The strength of synthetic promoters depends on the selection, spacing of CREs and the copy number. Using bioinformatic algorithms, novel CREs could be discovered, by comparing the upstream sequence of differentially regulated genes. These CREs could be put together rationally to design new synthetic promoters (Roberts 2011). Furthermore, the strength of the synthetic promoter could be enhanced by proper spacing and increasing the CRE copy number (Liu and Stewart 2016).

Here, we have tested three synthetic promoters in $P$. patens, developed using an automated highthroughput screening method. The promoters were built using computational analysis of large transcriptomic functional data set to identify cis-elements, which form the building blocks of synthetic promoter libraries. All three synthetic promoters had higher mRNA expression than the housekeeping gene PpAct7 and showed similar protein expression pattern to AtUBQ10 promoter. 
Promoter construction

84 The synthetic promoter library was constructed at Synpromics using random assembly techniques of 85 cis-elements through expression data analysis of Zea mays. Genes showing strong expression strength above Ubiquitinl transcripts were labeled constitutive. Using transcription factor binding site database TRANSFAC, cis-elements of the constitutively expressed genes were identified from the 1,500bp upstream and 500bp downstream of the transcription start site. Subsequently, Synpromics Ltd Syn-score algorithm was applied to the identified regions to rank the cis-elements (Roberts et al. 2017). Further, a synthetic promoter library was constructed by, random assembly of the chosen ciselements (300bp-800 bp), attached upstream to CaMV 35S minimal promoter (position -46 to +89 ). Later, functional promoters were identified by the expression analysis of the Luciferase gene.

Growth media at $23^{\circ} \mathrm{C}$.

DNA preparation and transformation protocol

DNA fragments for transformation were prepared in blocks as below. First block, a $2.7 \mathrm{~kb}$ region with 108 5' neutral locus, G418 selection marker with CaMV 35S promoter/ CaMV poly(A) signal was amplified from the pRH004 plasmid. Second block, the synthetic promoter sequences developed by Synpromics, and the Arabidopsis Ubiquitin10 (AtUBQ10) promoter was amplified with $20 \sim 22 \mathrm{nt}$ overhangs homologous to block one and three. Block 3, with the Venus fluorescent protein, OCS 
106 plasmid (Figure 1A). Purified 1.5 pmol of each DNA block (Figure 1B) was transfected into the 107 isolated moss protoplasts during transformation process and selected for positive colonies according 108 to previously published methods (King et al. 2016; Khairul Ikram et al. 2017).

110 Arabidopsis Ubiqutin10 promoter

111 Arabidopsis UBQ10 promoter with a length of $634 \mathrm{bp}$ of (Grefen et al. 2010) was cloned from 112 Arabidopsis genomic DNA using primer set; UBQ10 F 5'-GTCGACGAGTCAGTAATAAACGG113 3' and UBQ10 R 5'-CTGTTAATCAGAAAAACTCAGATTAATC-3'. For moss transformation, 22114 nt overhangs that are identical to the next fragments (block one and three) were attached to both ends 115 by second PCR using overhang primers.

\section{Detection of Venus fluorescence}

118 Venus fluorescence was detected on protonemal cells grown for seven days in PhyB liquid media. $P$. 119 patens protonema cells were visualized and photographed using a confocal laser-scanning 120 microscope. Z-stacks were performed on each line using the 488nm laser line and YFP emission 121 filter. Z-stacks were put together using the Zeiss software built-in maximum projection function. 122 Fluorescent level of each promoter lines was calculated from digital Images using the software 123 ImageJ (https://imagej.nih.gov/ij/). A previously published method on fluorescent cell analysis was 124 used to calculate the corrected total cell fluorescence (CTCF) levels (Burgess et al. 2010; McCloy et 125 al. 2014).

126 For each cell, measurements were taken for the cell area, integrated density and mean grey value.

127 Final corrected total cell fluorescence (CTCF) was calculated using the following formula. 
131 Total RNA was extracted from the appropriate lines (7 days after blending), using Spectrum ${ }^{\mathrm{TM}}$ Plant 132 Total RNA Kit (Sigma, STRN250). To synthesize cDNA, $1 \mu$ g of extracted total RNA was reverse 133 transcribed by iScript cDNA synthesis kit (Bio-rad, 1708891), followed by PCR amplification of the 134 following transcripts, PpAct7 and Venus. PCR reactions were carried out using (Qiagen kit name) 135 and (Bio-rad machine name), by denaturation at $95^{\circ} \mathrm{C} 5 \mathrm{~min}, 40$ cycles with $95^{\circ} \mathrm{C}$ for $10 \mathrm{sec}$ and $60^{\circ} \mathrm{C}$ 136 for $10 \mathrm{sec}$, and melting curve analysis to check the specificity. Relative Venus gene expression from 137 each promoter line was analyzed by Expvenus $=2^{\Delta \mathrm{Ct}[\text { promoter }]}, \Delta \mathrm{C}_{\mathrm{t}}[$ promoter $]=\mathrm{C}_{\mathrm{t}}\left[\right.$ Actin]- $\mathrm{C}_{\mathrm{t}}[$ Venus $]$. 


\section{Results and discussion}

140 Assembly of cis-elements to construct constitutive plant promoters

141 To generate synthetic promoters, functional cis-elements should be collected since $c i s$-elements will 142 form the building blocks of synthetic promoters. We used automated high-throughput screening 143 method. In this method, computational analysis of large transcriptomic functional data sets of Zea 144 mays was used to identify cis-elements from constitutively expressed genes. We ranked collected cis145 elements by applying Syn-score algorithm and randomly assembled selected cis-elements to generate 146 promoter library (Roberts et al, 2017). This technique has an advantage since elements are selected 147 based on the requirements for the synthetic promoters (e.g., inducible, constitutive and tissue- or 148 developmental stage-specific), which is a more focused approach than using completely random 149 elements. The promoter candidates consist of randomly assembled cis-elements of varying lengths 150 up to $30 \mathrm{bp}$. Therefore, the promoter length and the position of cis-elements vary (Figure 1C). It has 151 been shown that the position of cis-elements relative to each other markedly influences promoter 152 strength (Rushton et al. 2002), which was shown in the transcription data of the synthetic promoters 153 we tested (Figure 2).

154 The synthetic promoters can be used for gene stacking in P. patens, as they do not resemble each 155 other on the sequence level and contain small cis-elements rather than large promoter fragments, 156 which greatly reduces the risk of homology-induced gene silencing. As it is an automated system, it 157 is reproducible, robust and faster than a manual approach. Thus, multiple functional promoters in $P$. 158 patens could be constructed on demand.

Promoter activity analysis of synthetic promoters

161 To analyze the activity of synthetic promoters, we used fluorescence protein Venus. The coding sequence of Venus was placed downstream of each promoter including well-known constitutive 
AtUBQ10 promoter, and all constructs were stably transfected to P. patens 108 neutral locus (Bach et al. 2014). We performed qPCR to compare the promoter activity. The mRNA expression level of Venus was calculated in relative to the endogenous housekeeping gene Actin7 (PpAct7). Actin is an essential component of the plant cytoskeleton and is known to be a ubiquitous protein that is constitutively expressed in eukaryotes (Meagher et al. 1999). It is also shown that PpAct7 is preferable to be used as a housekeeping gene (Le Bail et al. 2013) and perform dual functions as a control and a housekeeping gene in the data analysis of this experiment. Expression of human VEGF protein using the $5^{\prime}$ promoter region of the PpAct 7 depicted an eight-fold increase in the production of the VEGF protein compared to the constitutive CaMV 35S promoter (Weise et al. 2006). This suggests $P p A c t 7$ is highly expressed compared to the $35 \mathrm{~S}$ promoter that was quantified previously (Horstmann et al. 2004). Thus, the mRNA expression of PpAct7 can be compared to the Venus transcript levels driven by the synthetic promoters. All three synthetic promoters showed higher expression than PpAct7 (Figure 2). Synthetic promoter I2-10 yielded the highest mRNA expression level at $304.51 \pm 4.21$ fold relative to PpAct7 followed by $45.91 \pm 0.88,124.93 \pm 3.4,258.38 \pm 0.09$ for $I 2-48, I 2-79$, and $A t U B Q 10$, respectively. The $I 2-10$ promoter was $2.43 \mathrm{X}$ and $6.63 \mathrm{X}$ expressed than I2-79 and I2-48. This high level of expression can be attributed to the presence of G-box in I210 (Figure 1A). The G-box is a regulatory element in plant promoters, playing an essential role in plant promoter responsiveness to light, stress, and hormones (Menkens et al. 1995). Some of the Gbox motifs have been shown to aid high-level constitutive protein expression in some plant species (Ishige et al. 1999).

Protein expression analysis of synthetic promoters in P. patens protonema cells After mRNA expression analysis, we wanted to identify if the protein expression would show similar pattern, because protein level does not always correspond to that of mRNA. The Venus protein 
expression level was calculated for each synthetic promotor line. This was performed in 14 days old P. patens protonema cells (Figure 3). Micrographs of I2-10, I2-48, I2-79, and AtUBQ10 promoter lines were processed to measure the fluorescence intensity of Venus via ImageJ. All three synthetic promoters showed a similar level of Venus fluorescent protein expression (Figure 3). Compared to the medium-strength of the AtUBQ10 promoter (Grefen et al. 2010), the synthetic promoters, $I 2-10, I 2-48$ and $I 2-79$ displayed $1.6 \mathrm{X}, 1.5 \mathrm{X}$ and $1.5 \mathrm{X}$ decrease in protein expression, respectively. All synthetic promoters share the identical sequence $-123 \mathrm{bp}$ from ATG, thus we excluded the possibility of ribosome entry for the discrepancy in expression of mRNA and protein level, and assume structure-related factor made the difference. These synthetic promoter candidates were derived from a screen conducted in Zea Mays, where Synpromics has shown that they mediate two- four-fold higher protein expression (firefly luciferase) compared to the ubiquitin-1 promoter. As one would expect we have seen that the promoters show maximum activity in the organism in which they were screened. The fact that promoters developed for another plant species show such high activity in P. patens, bodes well for the further development of promoters for use in this chassis organism.

The short length of 634 bp makes the $A t U B Q 10$ promoter an ideal control. However, the $A t U B Q 10$ promoter consisted of the first 5' intron expanding $304 \mathrm{bps}$, while synthetic promoters contained none. Deletion of the first 5' intron in $A t U B Q 10$ has shown to result in 3-fold lower protein activity than the first intron intact (Norris et al. 1993). Thus, adding an either synthetic or natural first 5' intron section to the synthetic promoters would likely lead even higher expression of the following 207 coding sequences.

In conclusion, we have tested the activity of three synthetic promoters in P. patens. All three promoters showed high expression of mRNA compared to the PpAct7 and similar protein activity to 
211 the medium-strength $A t U B Q 10$ promoter. Previously, published works have revealed that the addition 212 of the first $5^{\prime}$ intron increase the stability of the mRNA and yield several folds of higher protein 213 activity. Thus, adding a $5^{\prime}$ intron to the end of synthetic promoters would likely increase the strength 214 of the promoters. Further, range of promoters mediating a range of different expression levels is 215 essential in building genetic circuitry in synthetic biology applications, such that synthetic promoters 216 can control the correct stoichiometry of different component proteins of the circuit at the 217 transcriptional level. 


\section{Reference}

Bach SS, King BC, Zhan X, Simonsen HT, Hamberger B (2014) Plant Isoprenoids. 1153, 257-271. doi:10.1007/978-1-4939-0606-2.

Le Bail A, Scholz S, Kost B (2013) Evaluation of Reference Genes for RT qPCR Analyses of Structure-Specific and Hormone Regulated Gene Expression in Physcomitrella patens Gametophytes. PLoS ONE 8, 1-10. doi:10.1371/journal.pone.0070998.

Burgess A, Vigneron S, Brioudes E, Labbé J-C, Lorca T, Castro A (2010) Loss of human Greatwall results in G2 arrest and multiple mitotic defects due to deregulation of the cyclin B-Cdc2/PP2A balance. Proceedings of the National Academy of Sciences 107, 12564-12569. doi:10.1073/pnas.0914191107.

Grefen C, Donald N, Hashimoto K, Kudla J, Schumacher K, Blatt MR (2010) A ubiquitin-10 promoter-based vector set for fluorescent protein tagging facilitates temporal stability and native protein distribution in transient and stable expression studies. Plant Journal 64, 355-365. doi:10.1111/j.1365-313X.2010.04322.x.

Halpin C (2005) Gene stacking in transgenic plants - The challenge for 21 st century plant biotechnology. Plant Biotechnology Journal 3, 141-155. doi:10.1111/j.14677652.2004.00113.x.

Horstmann V, Huether CM, Jost W, Reski R, Decker EL (2004) Quantitative promoter analysis in Physcomitrella patens: A set of plant vectors activating gene expression within three orders of magnitude. BMC Biotechnology 4, 1-13. doi:10.1186/1472-6750-4-13.

Ikram NKBK, Zhan X, Pan X-W, King BC, Simonsen HT (2015) Stable heterologous expression of biologically active terpenoids in green plant cells. Frontiers in Plant Science 6,. doi:10.3389/fpls.2015.00129.

Ishige F, Takaichi M, Foster R, Chua NH, Oeda K (1999) A G-box motif (GCCACGTGCC) tetramer confers high-level constitutive expression in dicot and monocot plants. Plant Journal 18, 443448. doi:10.1046/j.1365-313X.1999.00456.x.

Khairul Ikram NKB, Beyraghdar Kashkooli A, Peramuna AV, van der Krol AR, Bouwmeester H, Simonsen HT (2017) Stable Production of the Antimalarial Drug Artemisinin in the Moss Physcomitrella patens. Frontiers in Bioengineering and Biotechnology 5, 1-8. doi:10.3389/fbioe.2017.00047.

King BC, Vavitsas K, Ikram NKBK, Schrøder J, Scharff LB, Hamberger B, Jensen PE, Simonsen HT (2016) In vivo assembly of DNA-fragments in the moss, Physcomitrella patens. Scientific Reports 6, 1-8. doi:10.1038/srep25030.

Latchman DS (1997) Transcription factors: An overview. International Journal of Biochemistry and Cell Biology 29, 1305-1312. doi:10.1016/S1357-2725(97)00085-X.

Liu W, Stewart CN (2016) Corrigendum to 'Plant synthetic promoters and transcription factors' [Curr. Opin. Biotechnol. 37 (2016) 36-44] doi 10.1016/j.copbio.2015.10.001. Current Opinion in Biotechnology 38, 203. doi:10.1016/j.copbio.2016.03.017.

McCloy RA, Rogers S, Caldon CE, Lorca T, Castro A, Burgess A (2014) Partial inhibition of Cdk1 in G2phase overrides the SAC and decouples mitotic events. Cell Cycle 13, 1400-1412. doi: $10.4161 / \mathrm{cc} .28401$.

Meagher RB, McKinney EC, Kandasamy MK (1999) Isovariant dynamics expand and buffer the responses of complex systems: the diverse plant actin gene family. The Plant cell 11, 995-1006. doi:10.1105/tpc.11.6.995.

Menkens AE, Schindler U, Cashmore AR (1995) The G-box: a ubiquitous regulatory DNA element in plants bound by the GBF family of bZIP proteins. Trends in Biochemical Sciences 20, 506510. doi:10.1016/S0968-0004(00)89118-5. 
Norris SR, Meyer SE, Callis J (1993) The intron of Arabidopsis thaliana polyubiquitin genes is conserved in location and is a quantitative determinant of chimeric gene expression. Plant Molecular Biology 21, 895-906. doi:10.1007/BF00027120.

Pan XW, Han L, Zhang YH, Chen DF, Simonsen HT (2015) Sclareol production in the moss Physcomitrella patens and observations on growth and terpenoid biosynthesis. Plant Biotechnology Reports 9, 149-159. doi:10.1007/s11816-015-0353-8.

Porto MS, Pinheiro MPN, Batista VGL, Dos Santos RC, De Albuquerque Melo Filho P, De Lima LM (2014) Plant promoters: An approach of structure and function. Molecular Biotechnology 56, 38-49. doi:10.1007/s12033-013-9713-1.

Rensing SA, Ick J, Fawcett JA, Lang D, Zimmer A, Van De Peer Y, Reski R (2007) An ancient genome duplication contributed to the abundance of metabolic genes in the moss Physcomitrella patens. BMC Evolutionary Biology 7, 1-10. doi:10.1186/1471-2148-7-130.

Roberts ML (2011) The Use of Functional Genomics in Synthetic Promoter Design. 'Comput. Biol. Appl. Bioinforma.' (Eds HS Lopes, LM Cruz)(InTech: Rijeka) doi:10.5772/20653.

Roberts ML, Katsoupi P, Tseveleki V, Taoufik E (2017) Bioinformatically Informed Design of Synthetic Mammalian Promoters. 'Mamm. Synth. Promot.' (Ed D Gould) pp. 93-112. (Springer New York: New York, NY) doi:10.1007/978-1-4939-7223-4_8.

Rushton P, Rushton PJ, Reinstädler A, Lipka V, Lippok B, Somssich IE (2002) Synthetic plant promoters containing defined regulatory elements provide novel insights into pathogen- and wound-induced signaling Synthetic Plant Promoters Containing Defined Regulatory Elements Provide Novel Insights into Pathogen- and Wound-Induced Sig. 14, 749-762. doi:10.1105/tpc.010412.can.

Sabovljević MS, Sabovljević AD, Ikram NKK, Peramuna A, Bae H, Simonsen HT (2016) Bryophytes - An emerging source for herbal remedies and chemical production. Plant Genetic Resources: Characterisation and Utilisation 14, 314-327. doi:10.1017/S1479262116000320.

Saidi Y, Finka A, Chakhporanian M, Zrÿd JP, Schaefer DG, Goloubinoff P (2005) Controlled expression of recombinant proteins in Physcomitrella patens by a conditional heat-shock promoter: A tool for plant research and biotechnology. Plant Molecular Biology 59, 697-711. doi:10.1007/s11103-005-0889-z.

Vesty EF, Saidi Y, Moody LA, Holloway D, Whitbread A, Needs S, Choudhary A, Burns B, McLeod D, Bradshaw SJ, Bae H, King BC, Bassel GW, Simonsen HT, Coates JC (2016) The decision to germinate is regulated by divergent molecular networks in spores and seeds. New Phytologist 211, 952-966. doi:10.1111/nph.14018.

Weise A, Rodriguez-Franco M, Timm B, Hermann M, Link S, Jost W, Gorr G (2006) Use of Physcomitrella patens actin 5\{textasciiacutex\} regions for high transgene expression: importance of 5 \{ttextasciiacutex introns. Applied Microbiology and Biotechnology 70, 337345. doi:10.1007/s00253-005-0087-6.

Wiedemann G, van Gessel N, Köchl F, Hunn L, Schulze K, Maloukh L, Nogué F, Decker EL, Hartung F, Reski R (2018) RecQ helicases function in development, DNA repair, and gene targeting in Physcomitrella patens. The Plant Cell tpc.00632.2017. doi:10.1105/tpc.17.00632.

Zhan X, Zhang Y-H, Chen D-F, Simonsen HT (2014) Metabolic engineering of the moss Physcomitrella patens to produce the sesquiterpenoids patchoulol and $\hat{\mathrm{I}} \pm / \hat{\mathrm{I}}^{2}$-santalene. Frontiers in Plant Science 5, 1-10. doi:10.3389/fpls.2014.00636. 


\section{$310 \quad$ Figure text}

311 Figure 1: A) vector map of linearized pRH004 vector.

312 B) Three PCR fragments (blocks) were constructed to transform the promoters to the P. patens 108 313 neutral loci. Block 1 was amplified from the pRH004 vector with the 108 neutral locus and resistance 314 to Geneticin driven by CaMV 35S promoter. Block 2 depicts, PCR amplified AtUBQ10, I2-10, I2-48 315 and I2-79 promoter sequences. Followed by the final block 3, containing the Venus fluorescent 316 protein with the OCS terminator sequence and the 108 loci.

317 C) Schematic of randomly assembled cis regulatory elements to construct the synthetic promoters.

319 Figure 2: Overview of promoter strength based on Venus expression, using PpAct7 promoter as 320 background measurement.

322 Figure 3: Confocal images of Venus expression, driven by synthetic promoters and AtUBQ10 323 promoter. All lines are grown and imaged in identical conditions. B) Representative, promoter 324 strengths of $I 2-10(\mathrm{n}=54), I 2-48(\mathrm{n}=41), I 2-79(\mathrm{n}=48)$ and $\operatorname{AtUBQ} 10(\mathrm{n}=32)$ lines. Promoter strength 325 was calculated by measurement of the fluorescence intensity for each cell by the use of confocal 326 micrographs and ImageJ software. Scale bar $=0.01 \mathrm{~mm}$ 